\title{
Wireless Micro-Sensor Network Models
}

\author{
Ms. Swapnali S. Maske ${ }^{1}$, Mrs. Tejaswini A. Pawar ${ }^{2}$ \\ ${ }^{I}$ Department of Information Technology, BVCOE, Shivaji University, Kolhapur, India. \\ ${ }^{2}$ Department of Computer Science and Engineering, BVCOE, Shivaji University, Kolhapur, India.
}

\begin{abstract}
Wireless sensor network is important in sensing, Collecting and disseminating information about environmental Phenomenon. This paper contain emerging field to classify wireless micro sensor network according to different communication functions, data delivery models and network dynamics. This taxonomy will aid in defining appropriate communication infra structure for diff. sensor network application subspaces. It allows network designer to choose the Protocol architecture. According to their application this taxonomy will enable new sensor network models to define for use in further research in this area. The overall communication behavior in a wireless micro sensor Network is application driven. It is useful to decouple the application communication used for information dissemination from the infrastructure communication used to configure and optimize the Network. This separation will aid network designers in selecting the appropriate sensor network architecture that will best match the characteristics of the communication traffic of a given application. This will allow the network protocol to achieve the application-specific goals of energy-efficiency, low latency, and high accuracy in the sensing application. We also believe that a sensor-initiated proactive path recovery approach with local patching will be beneficial in efficient information dissemination in wireless micro-sensor networks. The taxonomy presented will be helpful in designing and evaluating future network protocols for wireless micro-sensor networks. Often, it is possible to implement a sensor network for a specific phenomenon in a number of different ways. Consider the problem of monitoring a tornado. One option would be to fly airplanes to sense the tornado (mobile phenomenon; mobile sensors; continuous data delivery). Another would be to have a sensor grid statically placed on the ground and report data as the tornado passes through (mobile phenomenon; static sensors; continuous data delivery). Yet another would be to release lightweight sensors into the tornado (static phenomenon; mobile sensors; Continuous data delivery).
\end{abstract}

\section{Introduction}

Development in hardware and wireless network technology introduce a new area were small wireless devices will provide access to information any time, anywhere as well as actively participate in creating smart environment. Sensor networks hold the promise of revolutionizing sensing a wide range of application domains. This is because of their reliability, accuracy, flexibility, cast -effectiveness and case of development. Sensor are rapidly deployed in remote inhospitable area for a surveillance application sensors are used to analyze the motion of a tornado; Sensors are used in forest for fire detection; sensors are attached to tool cabs in a large metropolitan area to study the traffic conditions and plan routes effectively. There is wide range of applications for sensor network with differing requirements. In report the classification wireless micro-sensor networks from a communication protocol perspective, I look at characteristic and goals of typical micro-sensor network as well as different types of communication that required achieving their goals.

The remaining report is organized as Chapter 2 contains Performance Metrics of Sensor Network. Chapter 3 contains Architecture of Sensor Network. Chapter 4 contains The Data Delivery Models. Chapter 5 contains the Network Dynamic Models for Sensor Network. Chapter 6 contains Some Network Protocol, Following are the terminologies used in the report.

\subsection{Sensor}

The device that implements the physical sensing of environmental phenomena and reporting of measurements (through wireless communication). Typically it consists of five Components-sensing hardware, memory battery, embedded processor, and trance-receiver [1]. The basic sensor network is shown in Figure 1.1. 


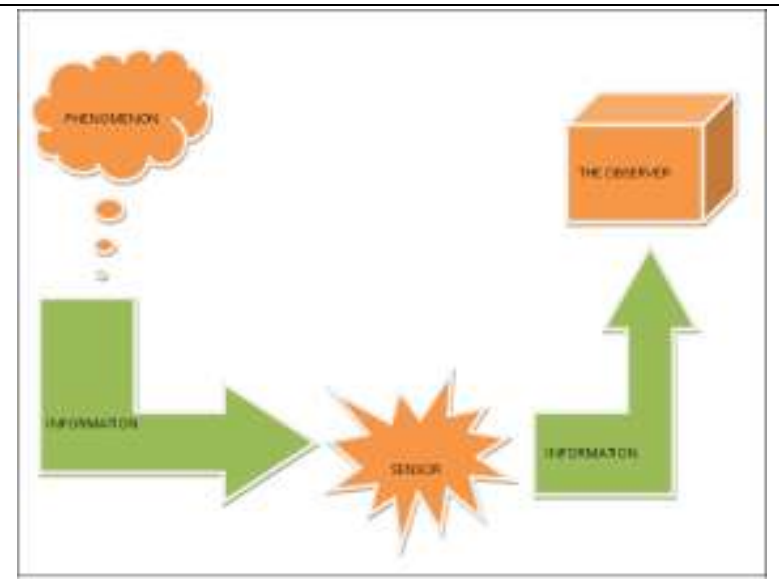

Fig. 1.1 The basic sensor network

\subsection{Observer}

The end user interested in obtaining information disseminated by the sensor network about the phenomena.

The observer may indicate interests (or queries) to the network and receive response to these queries [2].

\subsection{Phenomenon}

The entity of interest to the observer that is being served and optionally analyzed/filtered the sensor networks. There may be multiple phenomena under observation concurrently in the same network.

While monitoring the phenomena some latency and accuracy must be restricted. In a typical sensor network individuals sensor serve the local values and disseminate information to other sensors and to the observer. Sensors network shares many challenges of traditional wireless network. This include limited energy available to each node band width-limited, error-prone channel communication in sensor network is end-to-end. The function of network is to repeat information about phenomenon to observer. The observer not necessarily aware of the sensor networks infrastructure and individual sensor as end point communication.

\section{Performance Metrics}

Following are the metrics to evaluate sensor network protocols.

\subsection{Energy Efficient}

As sensor nodes are battery operated, protocols must be energy efficient or maximize system lifetime

\subsection{Scalability}

It is also a critical footer for large-scale network it is likely that locating interactions through hierarchy and aggregation will be critical for ensuring scalability.

\subsection{System Lifetime}

System lifetime is the time until half of nodes die or by application directed metrics such as when the network stop providing the application with desired information about phenomena.

\subsection{Latency}

The observer is interested in knowing about the phenomena within a given delay. The precise semantics of latency are application dependent.

\subsection{Accuracy}

Primary object of observer is to obtain the accurate information, where accuracy is determined by given application. The given infrastructure should be adaptive that the application obtain desired accuracy and latency with minimum energy used.

\subsection{Fault tolerance}

Due to surrounding physical conditions the sensor may fail, or also fails because of their energy ran out. This is difficult to replace the existing sensor, so the network is fault tolerance so that actual network condition is transparent to given application. 


\section{Sensor Network Architecture}

A Sensor network is a tool for measuring and relaying information about the phenomena to the observer within the desired performance bond and deployment cost. The organization of the network as shown in following Figure 3.1

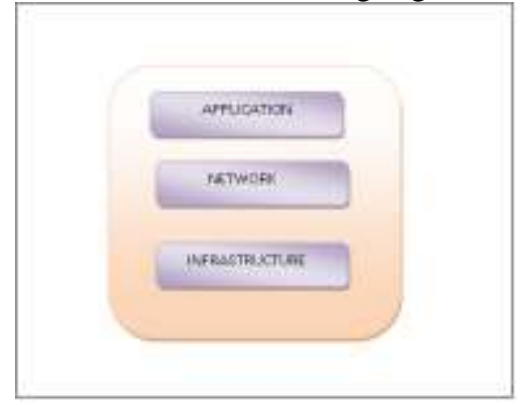

3.1 Infrastructure

Fig. 3.1 Sensor network architecture

The infrastructure consist of the sensor and their deployment status [2], more precisely it consist of characteristics of sensor e.g. sensing accuracy, memory size, battery life, transmission ranges and deployment strategy (e.g. sensor density, sensor location and mobility.)

\subsection{Network Protocols}

The network protocol is responsible creating paths and accomplishing communication between the sensors and observation

\subsection{Application / Observer}

The observer interest queries about the phenomenon as approximated by distributed data that the sensor is capable of serving.

These queries could be static or dynamic. The translation of data could be done by the application software at the observer and /or the sensor nodes or directed by human observer.

The network protocol in a sensor network is responsible for all communication both among sensors as well as between the sensors and observer.

In order to determine how the network protocol behaves for diff. scenario's, it is important to classify their feature. In next session different types of communication required in sensor network.

\subsection{Communication models}

The communication within a sensor network can be classified in to two-category application and infrastructure [1].

Application communication relates to the transfer of sensor data with the goal of informing the observer about the phenomena within application communication, there are two modes co-operative and non cooperative.

Non co-operative sensors do not cooperate at the application revel for information transfer.

The extreme case will be when no sensor communication with its neighbors. All sensors were independently. Co-operative sensors might be required to communicate with its neighbor $\mathrm{s}$ either periodically or after the occurrence of specific event.

Infrastructure communication refers to the communication needed to configure, maintain and optimize operation. The infrastructure communication is needed to keep the network functional ensure robust operation in dynamic environment as well as optimize overall performance.

A sensor network requires both the application and infrastructure communication. In static sensor networks an initial phase of infrastructure communion is needed to set up the networks furthermore, if the sensors are mobile, additional communications needed for path discovery/ reconfiguration.

\section{Data Delievery Models}

Sensor networks can be classified in terms of data delivery models required by applications (observer) interest as

1.Continues

2. Event driven

3.Observer initiated

4. Hybrid

4.1 Continues 
In the Continues models the sensor communicates their data continuously at a pre-specified rate [1] [2]. Clustering is the most efficient for static networks where data is continuously transmitted.

\subsection{Event Driven}

In the event driven data models the sensors reporting information only if an event of interest occurs, here observer is interested in the occurrence of a specific phenomenon or a set of phenomenon [1] [2].

\subsection{Observer Initiated}

In the observer initiated (or request reply) model the sensors only report their results in response to an explicit request from the observer (either directly, or indirectly through other sensors)

\subsection{Hybrid}

Hybrid models are one where the above three approaches can co exists in the network.

In this way we have only classify the data delivery from the application perspective. For any of the above models the communication approaches can be classified as 1. Flooding (Broadcast based) 2. Unicast 3 . Multicast. In flooding approach sensor broadcast information in neighboring node and it rebroadcast this data until reaches to the observer.

\section{Network Dynamics Models}

A path is formed between phenomena and the observer by a sensor network the sensor network protocol creates and maintains this path (or multiple paths) under dynamic condition while meeting the application requirements of low energy, low latency, high accuracy and fault tolerance.

There are several approaches to construct and maintain path between the observer and the phenomenon. They are classified as static sensor network and mobile / dynamic sensor network, the important point is mobility because it is most common source of dynamic conditions other source includes sensors failure and change in observer interest.

\subsection{Static Sensor Network}

There is no motion among communication sensors, the observer and the phenomenon. An example is a group of sensors spread for temperature sensing.

In this type of network, sensor nodes requires an initial one time setup infrastructure communication to create the path between the observer and the sensors with the remaining traffic exclusively application communication.

\subsection{Dynamic Sensors Network}

In dynamic sensor network, either the sensors themselves, the observer or the phenomenon are mobile, whenever a phenomenon associated with sensors moves, the path between observer and phenomenon get failed, in such situation either observer or the sensor must rebuild a new path. There are two types of rebuilding of new paths between observer and sensors.

The first approaches is observer-initiated approach, in this case during initial setup the observer can build multiple paths between itself and the phenomenon. The observer can use the most beneficial path, if path fails another path can be used, if all paths fail, the observer must rebuild new paths. This observer initiated approach is a reactive approach where path recovery action is only taken after observe a broken path [1] [2].

Another approach is sensor-initiated approach. In sensor initiated path recovery procedure, path recovery is initiated by a sensor that is currently a part of logical path between the observer \& the phenomenon. It is planning to move out of range. The sensors perform some local patching by broadcasting a participation request to neighboring sensor for given logical flow to build a new path. If any sensor sends participation reply message then the new path is build, if none of sensor reply then sensor can default to sending a path invalidation request to the observation so that the observer can start building the path.

Dynamic sensor networks can be further classified by considering the motion of the components. This motion is important. Each of following requires different infrastructure; data delivery models and protocols

\subsubsection{Mobile observer}

In this case the observer is mobile with respect to the sensors and phenomena. An example of this paradigm is sensors deployed in an inhospitable area for environment monitoring. For example, a plane might fly over a field periodically to collect information from a sensor network. Thus the observer, in the plane, is moving relative to the sensors and phenomena on the ground. 


\subsubsection{Mobile sensors}

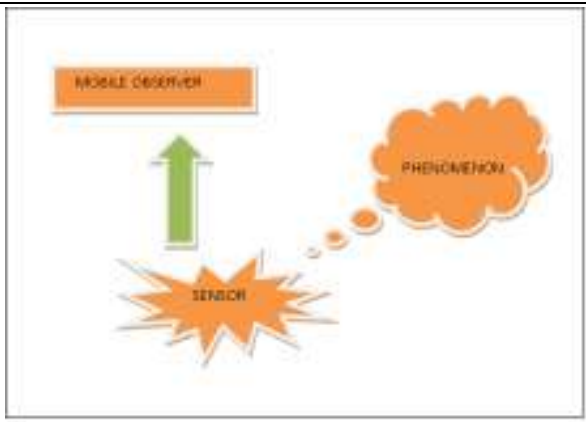

Fig. 5.2.1 Mobile observer

In this case, the sensors are moving with respect to each other and the observer. For example, consider traffic monitoring implemented by attaching sensors to taxis. As the taxis move, the attached sensors continuously communicate with each other about their own observations of the traffic conditions. If the sensors are co-operative, the communication paradigm imposes additional constraints such as detecting the link layer addresses of the neighbors and constructing localization and in formation dissemination structures. We know that the overhead of maintaining a globally unique sensor ID in a hierarchical fashion like an IP address is expensive and not needed. Instead, these sensors should communicate only with their neighbors with the link layer MAC address. In such networks, the above-mentioned proactive algorithm with local patching for repairing a path can be used so that the information about the phenomenon is always available to the observer regardless of the Mobility of the individual sensors.

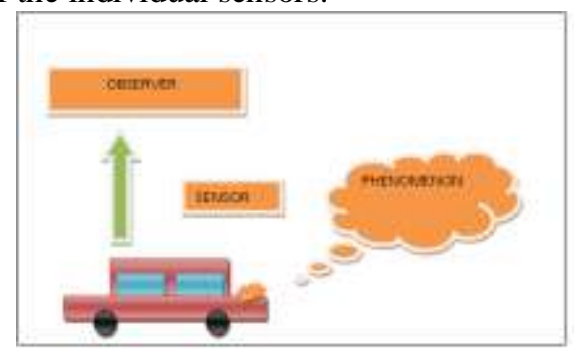

\subsubsection{Mobile Phenomena}

Fig. 5.2.2 Mobile sensor

In this case, the phenomenon itself is moving. A typical example of this paradigm is sensors deployed for animal detection. In this case the infrastructure level communication should be event-driven. Depending on the density of the phenomena, it will be inefficient if all the sensor nodes are active all the time. Only the sensors in the vicinity of the mobile phenomenon need to be active. The number of active sensors in the vicinity of the phenomenon can be determined by application specific goals such as accuracy, latency, and energy efficiency.

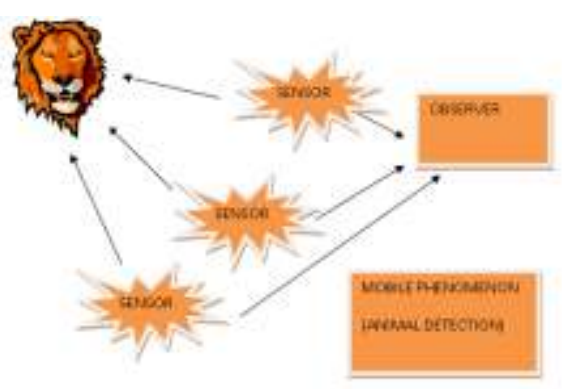

Fig 5.2.3 Mobile phenomenon

\section{Overview Of Some Network Protocols}

In this section we consider several existing protocols for sensor networks and analyze them in the context of our taxonomy. 
6.1 Ad Hoc Routing Protocol

Ad hoc routing protocols may be used as the network protocol for sensor networks [2]. However, such protocols will generally not be good candidates for sensor networks because of the following reasons:

(i) Sensors have low battery power and low available memory;

(ii) The routing table size scales with the network size;

(iii) These networks are designed for end-to-end communication and react inappropriately to mobility;

(iv) Their addressing requirements may be inappropriate for sensor networks;

(v) Ad hoc routing protocols do not support cooperative dissemination;

As ad hoc routing protocols do not inherently support data aggregation or fusion, they will not perform well in sensor network applications.

\subsection{LEACH}

$\mathrm{ACH}$ is an energy efficient protocol for sensor networks designed for sensor networks with continuous data delivery mechanism and no mobility [2]. LEACH uses a clustering architecture where member nodes send their data to the local cluster-head. Cluster-heads aggregate the data from each sensor and then send this information to the observer node. LEACH uses rotation of the cluster head in order to evenly distribute the energy load. Once clusters are formed, cluster members use TDMA to communicate with the cluster-head. Thus LEACH is suitable for networks where every node has data to send at regular intervals. However, it needs to be extended for event driven models as well as for mobile sensors.

\subsection{Direct Diffusion}

Directed Diffusion (DD) is a data-centric protocol, where nodes are not addressed by their addresses but by the data they sense [2]. Attribute-value pairs name data. In directed diffusion observer nodes express the interest in term of a query, which diffuses through the network using local interactions. Once a sensor node that satisfies the query (source node) is reached, that node starts transmitting data to the sink node, again using local interactions. The absence of a notion of a global id (e.g., IP address) makes directed diffusion efficient for networks with mobility as well. Directed diffusion is applicable for Event-driven and query-driven networks as defined in our taxonomy.

\section{Conclusion}

The overall communication behavior in a wireless micro sensor Network is application driven. It is useful to decouple the application communication used for information dissemination from the infrastructure communication used to configure and optimize the Network. This separation will aid network designers in selecting the appropriate sensor network architecture that will best match the characteristics of the communication traffic of a given application.

This will allow the network protocol to achieve the application-specific goals of energy-efficiency, low latency, and high accuracy in the sensing application. We also believe that a sensor-initiated proactive path recovery approach with local patching will be beneficial in efficient information dissemination in wireless micro-sensor networks.

The taxonomy presented will be helpful in designing and evaluating future network protocols for wireless micro-sensor networks.

Often, it is possible to implement a sensor network for a specific phenomenon in a number of different ways. Consider the problem of monitoring a tornado. One option would be to fly airplanes to sense the tornado (mobile phenomenon; mobile sensors; continuous data delivery). Another would be to have a sensor grid statically placed on the ground and report data as the tornado passes through (mobile phenomenon; static sensors; continuous data delivery). Yet another would be to release lightweight sensors into the tornado (static phenomenon; mobile sensors; Continuous data delivery).

The accuracy is a function of the sensing technology of the sensors and their distance from the phenomenon. However, since the performance is measured at the observer end, it is also a function of the performance of the communication model. We hope that this taxonomy will assist in developing relevant simulation models to enable empirical study of the performance of the different sensor network organizations and assist in making design and deployment decisions.

\section{References}

[1] Sameer Tilak, Nael B, Abu-Ghazaleh and Wendi Heizelmn,"A Taxonomy of Wireless Micro Sensor Network Models",

[2] Sameer Tilak, Nael B, Abu-Ghazaleh and Wendi Heizelmn ," The Updatesd A Taxonomy of Wireless Micro Sensor Network Models",ACM And Mobile Computing Review, April 2002,Volume 1,Number 2. 